\title{
Self-assembly of T-structures in Molecular Fluids
}

\author{
Amar B. Pawar and Ilona Kretzschmar* \\ Department of Chemical Engineering, City College of New York, $140^{\text {th }}$ street and Convent \\ Avenue, New York city, NY 10031, USA \\ Gregory Aranovich and Marc D. Donohue
}

Department of Chemical and Biomolecular Engineering, Johns Hopkins University, Baltimore, MD, USA

*CORRESPONDING AUTHOR Ilona Kretzschmar, kretzschmar@ccny.cuny.edu

\section{Details of the calculations}

The four-pole patchy particles can orient themselves in any of the four orientations on a lattice site $(i, j)$ illustrated in Figure S1. $x_{1, p, i, j}, x_{2, p, i, j}, x_{3, p, i, j}$, and $x_{4, p, i, j}$ are the

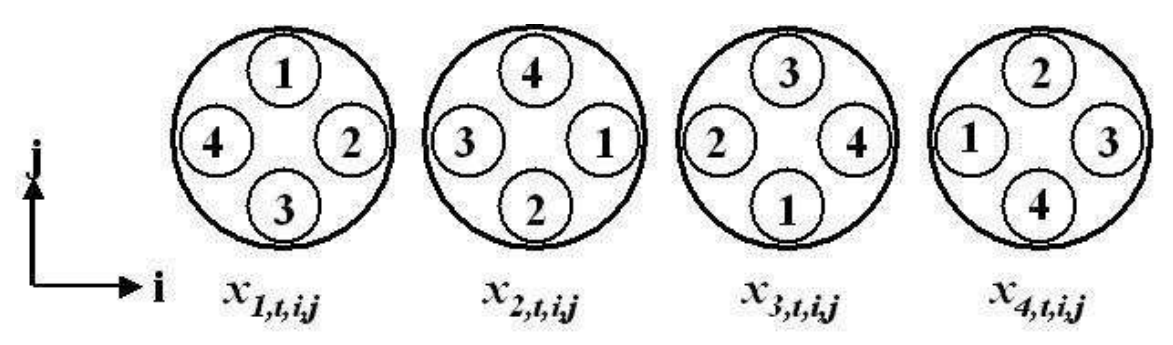

Fig.S1. The four orientations a four-pole particle can assume on each site of a planar lattice. $x_{k, t, i, j}\left(\mathrm{t}=\mathrm{A}, \mathrm{B}_{n}\right.$, or $\mathrm{C}$ and $\left.k=1-4\right)$ is the probability that the lattice site $(i, j)$ is occupied by a $t$ type particle with orientation $k$.

probabilities of finding a $p$ type particle $\left(p=A, B_{n}: n=1,2\right.$, or $\left.C\right)$ with a particular orientation at the lattice site $(i, j)$. The total density, $\rho(i, j)$, for a $p$ type particle at the site $(i, j)$ is given by:

$$
\rho_{p}(i, j)=x_{1, p, i, j}+x_{2, p, i, j}+x_{3, p, i, j}+x_{4, p, i, j}
$$

The configurational internal energy per particle resulting from two neighboring lattice sites has contributions from the interactions between the same types of particles $(A A$, 
$B_{n} B_{n}$, and $\left.C C\right)$ as well as between different types of particles $\left(A B_{n}, A C\right.$, and $\left.B_{n} C\right)$ and can be written as:

$$
E=\left(\frac{1}{2}\right) \sum_{a=\text { particle } 1}^{\text {particle3 }} \sum_{b=\text { particle } 1}^{\text {particle } 3} \sum_{m=\text { orientation } 1}^{\text {orientation } 4} \sum_{n=\text { orientation } 1}^{\text {orientation } 4}\left(\epsilon_{\text {abmn }} p(a, m) p(b, n)\right)
$$

where $\epsilon_{a b m n}$ is the interaction energy between the $a$ th and $b$ th type of particle $(a, b$ $=1-3)$ with the contact points $m$ and $n(m, n=1-4) \cdot p(a, m)$ and $p(b, n)$ are the respective probabilities of finding the $a$ th and bth type of particle on neighboring sites to have a contact point $m$ on the $a$ th type of particle and a contact point $n$ on the $b$ th type of particle. The total Hamiltonian thus can be written as:

$$
\begin{aligned}
& U=\left(\frac{1}{2}\right) \sum_{p=1}^{3} \sum_{q=1}^{3} \sum_{i=-i^{*}}^{i^{*}} \sum_{j=-j^{*}}^{j^{*}} \\
& \left(x_{1, p, i, j} x_{1, q, i+1, j} \epsilon_{p q 24}+x_{1, p, i, j} x_{2, q, i+1, j} \epsilon_{p q 23}+x_{1, p, i, j} x_{3, q, i+1, j} \epsilon_{p q 22}+x_{1, p, i, j} x_{4, q, i+1, j} \epsilon_{p q 21}\right. \\
& +x_{2, p, i, j} x_{1, q, i+1, j} \epsilon_{p q 14}+x_{2, p, i, j} x_{2, q, i+1, j} \epsilon_{p q 13}+x_{2, p, i, j} x_{3, q, i+1, j} \epsilon_{p q 12}+x_{2, p, i, j} x_{4, q, i+1, j} \epsilon_{p q 11} \\
& +x_{3, p, i, j} x_{1, q, i+1, j} \epsilon_{p q 44}+x_{3, p, i, j} x_{2, q, i+1, j} \epsilon_{p q 43}+x_{3, p, i, j} x_{3, q, i+1, j} \epsilon_{p q 42}+x_{3, p, i, j} x_{4, q, i+1, j} \epsilon_{p q 41} \\
& \left.+x_{4, p, i, j} x_{1, q, i+1, j} \epsilon_{p q 34}+x_{4, p, i, j} x_{2, q, i+1, j} \epsilon_{p q 33}+x_{4, p, i, j} x_{3, q, i+1, j} \epsilon_{p q 32}+x_{4, p, i, j} x_{4, q, i+1, j} \epsilon_{p q 31}\right) \\
& +\left(x_{1, p, i, j} x_{1, q, i-1, j} \epsilon_{p q 42}+x_{1, p, i, j} x_{2, q, i-1, j} \epsilon_{p q 41}+x_{1, p, i, j} x_{3, q, i-1, j} \epsilon_{p q 44}+x_{1, p, i, j} x_{4, q, i-1, j} \epsilon_{p q 43}\right. \\
& +x_{2, p, i, j} x_{1, q, i-1, j} \epsilon_{p q 32}+x_{2, p, i, j} x_{2, q, i-1, j} \epsilon_{p q 31}+x_{2, p, i, j} x_{3, q, i-1, j} \epsilon_{p q 34}+x_{2, p, i, j} x_{4, q, i-1, j} \epsilon_{p q 33} \\
& +x_{3, p, i, j} x_{1, q, i-1, j} \epsilon_{p q 22}+x_{3, p, i, j} x_{2, q, i-1, j} \epsilon_{p q 21}+x_{3, p, i, j} x_{3, q, i-1, j} \epsilon_{p q 24}+x_{3, p, i, j} x_{4, q, i-1, j} \epsilon_{p q 23} \\
& \left.+x_{4, p, i, j} x_{1, q, i-1, j} \epsilon_{p q 12}+x_{4, p, i, j} x_{2, q, i-1, j} \epsilon_{p q 11}+x_{4, p, i, j} x_{3, q, i-1, j} \epsilon_{p q 14}+x_{4, p, i, j} x_{4, q, i-1, j} \epsilon_{p q 13}\right) \\
& +\left(x_{1, p, i, j} x_{1, q, i, j+1} \epsilon_{p q 13}+x_{1, p, i, j} x_{2, q, i, j+1} \epsilon_{p q 12}+x_{1, p, i, j} x_{3, q, i, j+1} \epsilon_{p q 11}+x_{1, p, i, j} x_{4, q, i, j+1} \epsilon_{p q 14}\right. \\
& +x_{2, p, i, j} x_{1, q, i, j+1} \epsilon_{p q 43}+x_{2, p, i, j} x_{2, q, i, j+1} \epsilon_{p q 42}+x_{2, p, i, j} x_{3, q, i, j+1} \epsilon_{p q 41}+x_{2, p, i, j} x_{4, q, i, j+1} \epsilon_{p q 44} \\
& +x_{3, p, i, j} x_{1, q, i, j+1} \epsilon_{p q 33}+x_{3, p, i, j} x_{2, q, i, j+1} \epsilon_{p q 32}+x_{3, p, i, j} x_{3, q, i, j+1} \epsilon_{p q 31}+x_{3, p, i, j} x_{4, q, i, j+1} \epsilon_{p q 34} \\
& \left.+x_{4, p, i, j} x_{1, q, i, j+1} \epsilon_{p q 23}+x_{4, p, i, j} x_{2, q, i, j+1} \epsilon_{p q 22}+x_{4, p, i, j} x_{3, q, i, j+1} \epsilon_{p q 21}+x_{4, p, i, j} x_{4, q, i, j+1} \epsilon_{p q 24}\right) \\
& +\left(x_{1, p, i, j} x_{1, q, i, j+1} \epsilon_{p q 31}+x_{1, p, i, j} x_{2, q, i, j-1} \epsilon_{p q 34}+x_{1, p, i, j} x_{3, q, i, j+1} \epsilon_{p q 33}+x_{1, p, i, j} x_{4, q, i, j-1} \epsilon_{p q 32}\right. \\
& +x_{2, p, i, j} x_{1, q, i, j-1} \epsilon_{p q 21}+x_{2, p, i, j} x_{2, q, i, j-1} \epsilon_{p q 24}+x_{2, p, i, j} x_{3, q, i, j-1} \epsilon_{p q 23}+x_{2, p, i, j} x_{4, q, i, j-1} \epsilon_{p q 22} \\
& +x_{3, p, i, j} x_{1, q, i, j-1} \epsilon_{p q 11}+x_{3, p, i, j} x_{2, q, i, j-1} \epsilon_{p q 14}+x_{3, p, i, j} x_{3, q, i, j-1} \epsilon_{p q 13}+x_{3, p, i, j} x_{4, q, i, j-1} \epsilon_{p q 12} \\
& \left.+x_{4, p, i, j} x_{1, q, i, j-1} \epsilon_{p q 41}+x_{4, p, i, j} x_{2, q, i, j-1} \epsilon_{p q 44}+x_{4, p, i, j} x_{3, q, i, j-1} \epsilon_{p q 43}+x_{4, p, i, j} x_{4, q, i, j-1} \epsilon_{p q 42}\right)
\end{aligned}
$$


$i^{*}$ and $j^{*}$ indicate the lattice size. The lattice site $(0,0)$ is occupied by a three patch particle ( $A$ particle) with the orientation 1 (i.e. $x_{1, A, 0,0}=1$ ). The entropy of the system (per one site) in the mean field approximation can be written as:

$$
\begin{aligned}
S= & k_{B} \sum \sum_{(i, j) \neq(0,0)}\left\{\left[\sum _ { p = 1 } ^ { 3 } \left(x_{1, p, i, j} \ln x_{1, p, i, j}+x_{2, p, i, j} \ln x_{2, p, i, j}+x_{3, p, i, j} \ln x_{3, p, i, j}\right.\right.\right. \\
& \left.\left.+x_{4, p, i, j} \ln x_{4, p, i, j}\right)\right]+\left[1-\sum_{p=1}^{3}\left(x_{1, p, i, j}+x_{2, p, i, j}+x_{3, p, i, j}+x_{4, p, i, j}\right)\right] \\
& {\left.\left[\ln \left(1-\sum_{p=1}^{3}\left(x_{1, p, i, j}+x_{2, p, i, j}+x_{3, p, i, j}+x_{4, p, i, j}\right)\right)\right]\right\} }
\end{aligned}
$$

The free energy, $F$, can be obtained from Eqs. ( 3 ) and ( 4 ) as:

$$
F=U-T S
$$

where $T$ is the absolute temperature and $k_{B}$ is the Boltzmann's constant. Minimization of the functional $F$ under the constraint Eq. (6 ) will give the equilibrium density distributions of the particles.

$$
N \sum_{i=-i^{*}}^{i^{*}} \sum_{j=-j^{*}}^{j^{*}}\left(x_{1, p, i, j}+x_{2, p, i, j}+x_{3, p, i, j}+x_{4, p, i, j}\right)=N_{p}
$$

where $N$ is the total number of lattice sites and $N_{p}$ is the total number of $p$ type particle. Minimization under the constraint is done by the standard method of Lagrange's multipliers by considering a function:

$$
\Omega=F-\sum_{p=1}^{3}\left[\sum_{i=-i^{*}}^{i^{*}} \sum_{j=-j^{*}}^{j^{*}}\left(x_{1, p, i, j}+x_{2, p, i, j}+x_{3, p, i, j}+x_{4, p, i, j}\right)-N_{p} / N\right]
$$

which gives following the equations for Lagrange's multipliers, $\mu_{p}$ :

$$
\frac{\partial \Omega}{\partial x_{k, p, i, j}}=0
$$


where $k=1,2,3$, 4. Eq. ( 8 ) and Eqs. ( 3 ) - ( 5 ) give:

$$
E_{k, p}+k_{B} T \ln \left\{\frac{x_{k, p, i, j}}{1-\sum_{q=1}^{3}\left(x_{1, q, i, j}+x_{2, q, i, j}+x_{3, q, i, j}+x_{4, q, i, j}\right)}\right\}=\mu_{p}
$$

where the terms $E_{k, p}$ are defined by:

$$
\begin{aligned}
& E_{1, p}=\sum_{q=1}^{3}\left\{x_{1, q, i+1, j} \epsilon_{p q 24}+x_{2, q, i+1, j} \epsilon_{p q 23}+x_{3, q, i+1, j} \epsilon_{p q 22}+x_{4, q, i+1, j} \epsilon_{p q 21}\right. \\
& +x_{1, q, i-1, j} \epsilon_{p q 42}+x_{2, q, i-1, j} \epsilon_{p q 41}+x_{3, q, i-1, j} \epsilon_{p q 44}+x_{4, q, i-1, j} \epsilon_{p q 43} \\
& +x_{1, q, i, j+1} \epsilon_{p q 13}+x_{2, q, i, j+1} \epsilon_{p q 12}+x_{3, q, i, j+1} \epsilon_{p q 11}+x_{4, q, i, j+1} \epsilon_{p q 14} \\
& \left.+x_{1, q, i, j-1} \epsilon_{p q 31}+x_{2, q, i, j-1} \epsilon_{p q 34}+x_{3, q, i, j-1} \epsilon_{p q 33}+x_{4, q, i, j-1} \epsilon_{p q 32}\right\} \\
& E_{2, p}=\sum_{q=1}^{3}\left\{x_{1, q, i+1, j} \epsilon_{p q 14}+x_{2, q, i+1, j} \epsilon_{p q 13}+x_{3, q, i+1, j} \epsilon_{p q 12}+x_{4, q, i+1, j} \epsilon_{p q 11}\right. \\
& +x_{1, q, i-1, j} \epsilon_{p q 32}+x_{2, q, i-1, j} \epsilon_{p q 31}+x_{3, q, i-1, j} \epsilon_{p q 34}+x_{4, q, i-1, j} \epsilon_{p q 33} \\
& +x_{1, q, i, j+1} \epsilon_{p q 43}+x_{2, q, i, j+1} \epsilon_{p q 42}+x_{3, q, i, j+1} \epsilon_{p q 41}+x_{4, q, i, j+1} \epsilon_{p q 44} \\
& \left.+x_{1, q, i, j-1} \epsilon_{p q 21}+x_{2, q, i, j-1} \epsilon_{p q 24}+x_{3, q, i, j-1} \epsilon_{p q 23}+x_{4, q, i, j-1} \epsilon_{p q 22}\right\} \\
& E_{3, p}=\sum_{q=1}^{3}\left\{x_{1, q, i+1, j} \epsilon_{p q 44}+x_{2, q, i+1, j} \epsilon_{p q 43}+x_{3, q, i+1, j} \epsilon_{p q 42}+x_{4, q, i+1, j} \epsilon_{p q 41}\right. \\
& +x_{1, q, i-1, j} \epsilon_{p q 22}+x_{2, q, i-1, j} \epsilon_{p q 21}+x_{3, q, i-1, j} \epsilon_{p q 24}+x_{4, q, i-1, j} \epsilon_{p q 23} \\
& +x_{1, q, i, j+1} \epsilon_{p q 33}+x_{2, q, i, j+1} \epsilon_{p q 32}+x_{3, q, i, j+1} \epsilon_{p q 31}+x_{4, q, i, j+1} \epsilon_{p q 34} \\
& \left.+x_{1, q, i, j-1} \epsilon_{p q 11}+x_{2, q, i, j-1} \epsilon_{p q 14}+x_{3, q, i, j-1} \epsilon_{p q 13}+x_{4, q, i, j-1} \epsilon_{p q 12}\right\} \\
& E_{4, p}=\sum_{q=1}^{3}\left\{x_{1, q, i+1, j} \epsilon_{p q 34}+x_{2, q, i+1, j} \epsilon_{p q 33}+x_{3, q, i+1, j} \epsilon_{p q 32}+x_{4, q, i+1, j} \epsilon_{p q 31}\right. \\
& +x_{1, q, i-1, j} \epsilon_{p q 12}+x_{2, q, i-1, j} \epsilon_{p q 11}+x_{3, q, i-1, j} \epsilon_{p q 14}+x_{4, q, i-1, j} \epsilon_{p q 13} \\
& +x_{1, q, i, j+1} \epsilon_{p q 23}+x_{2, q, i, j+1} \epsilon_{p q 22}+x_{3, q, i, j+1} \epsilon_{p q 21}+x_{4, q, i, j+1} \epsilon_{p q 24} \\
& \left.+x_{1, q, i, j-1} \epsilon_{p q 41}+x_{2, q, i, j-1} \epsilon_{p q 44}+x_{3, q, i, j-1} \epsilon_{p q 43}+x_{4, q, i, j-1} \epsilon_{p q 42}\right\}
\end{aligned}
$$


The values of $\mu_{p}$ can be obtained from the requirement that all $x_{k, p, i, j}=x_{\infty, p} / 4$, as the distance from the center molecule goes to infinity. Thus Eq. ( 9 ) gives:

$$
E_{0, p}+k_{B} T \ln \left\{\frac{x_{\infty, p} / 4}{1-\sum_{q=1}^{3} x_{\infty, q}}\right\}=\mu_{p}
$$

where $E_{0, p}$ is obtained from Eqs. ( 10$)$ - ( 13$)$ and $x_{k, p, i, j}=x_{\infty, p} / 4$. Combining Eq. ( 9 ) and Eq. ( 14 ) results in:

$$
k_{B} T \ln \left\{\frac{x_{k, p, i, j}\left(1-\sum_{q=1}^{3} x_{\infty, q}\right)}{\left(1-\sum_{q=1}^{3} x_{1, q, i, j}+x_{2, q, i, j}+x_{3, q, i, j}+x_{4, q, i, j}\right)\left(x_{\infty, p} / 4\right)}\right\}+E_{k, p}-E_{0, p}=0
$$

which can be represented in the following form:

$$
x_{k, p, i, j}=\left\{\frac{x_{\infty, p} \exp \left[\frac{E_{0, p}-E k, p}{k_{B} T}\right]}{4\left(1-\sum_{q=1}^{3} x_{\infty, q}\right)+\sum_{q=1}^{3} \sum_{s=1}^{4} x_{\infty, q} \exp \left[\frac{E_{0, q}-E s, q}{k_{B} T}\right]}\right\}
$$

The set of equations given by Eq. ( 16 ) gives the equilibrium density distribution of particles. The non-linear simultaneous equations given by Eq. ( 16 ) are solved by the successive substitution method for lattice size of $i^{*}=j^{*}=10$ with periodic boundary conditions for different sets of parameters for Case 1 and Case 2. 


\section{Equilibrium density distribution plots}

Case 1: Symmetric two-patch particle

Figures S2(a) and S2(b) show the sample density distributions of $\mathrm{A}(*), \mathrm{B}_{1}(\mathrm{O})$, and $\mathrm{C}(\square)$ particles around an A particle at $(0,0)$ observed in the calculations, at two different attractive rest all interactions $\left(\epsilon_{\text {restall }}=0.01 \mathrm{eV}\right.$ and $\left.0.032 \mathrm{eV}\right)$, which correspond to the T-structure and non-specific condensation of particles respectively. The density distributions highlighted by Plane 1 which correspond to a density distribution of 1 , indicate that these lattice sites are occupied by particles.

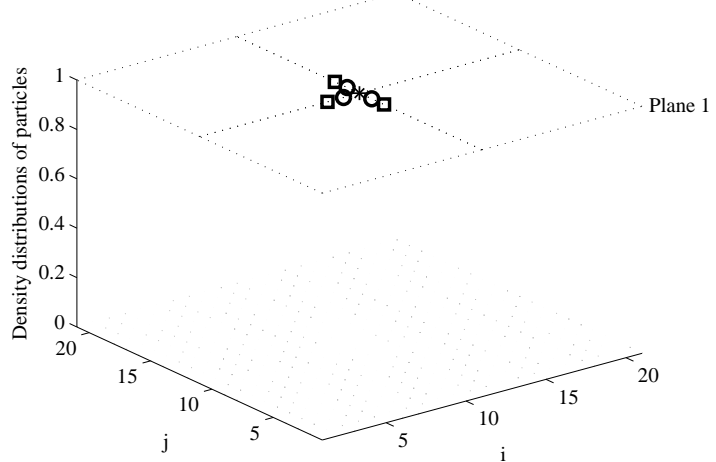

(a)

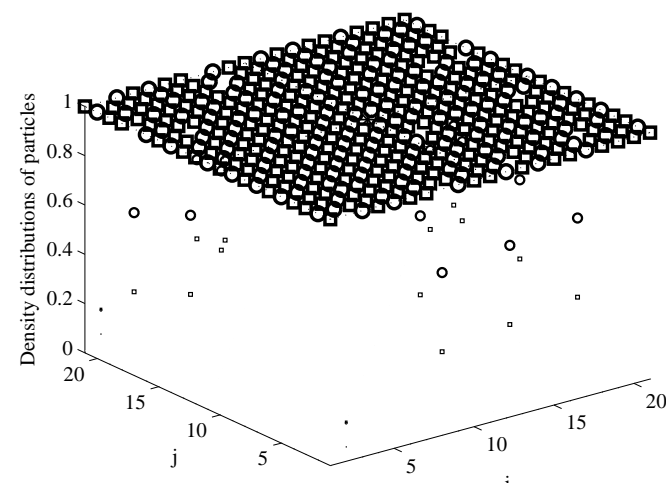

(b)

Fig.S2. Density plots of the equilibrium structures formed at $300 K$ in Case 1 with constant $\mathrm{A}\left(*, x_{\infty A}=0.001\right), \mathrm{B}_{1}\left(\mathrm{O}, x_{\infty B_{1}}=0.003\right)$, and $\mathrm{C}\left(\square, x_{\infty C}=0.003\right)$ particle concentrations and varying interaction energies. (a) T-structure at strong interaction energy $=-3 \mathrm{eV}$, weak interaction energy $=-0.5 \mathrm{eV}$ and rest all interaction energy $=-0.01 \mathrm{eV}$, (b) Condensed particles at strong interaction energy $=-3 \mathrm{eV}$, weak interaction energy $=-0.5 \mathrm{eV}$ and rest all interaction energy $=-0.032 \mathrm{eV}$.

\section{Case 2: Asymmetric two-patch particle}

Figures S3 (a)-(d) show sample density distributions obtained for A, $B_{2}$, and C particles around an $\mathrm{A}$ particle at $(0,0)$ at various $\mathrm{B}_{2}$ and $\mathrm{C}$ particle concentrations of (a) $x_{\infty B_{2}}=0.0005$ and $x_{\infty C}=0.004$, (b) $x_{\infty B_{2}}=0.00153$ and $x_{\infty C}=0.004$, (c) $x_{\infty B_{2}}=0.0004$ and $x_{\infty C}=0.004$, and $(\mathrm{d}) x_{\infty B_{2}}=0.0025$ and $x_{\infty C}=0.008$, while $x_{\infty A}$ is kept constant at 0.0001 which correspond to parameter sets leading to (a) a mixture of T-structures, (b) chains and an extended T-structure, (c) condensation of A particles, and (d) dimers of $\mathrm{C} / \mathrm{B}_{2}$ particles, respectively. 

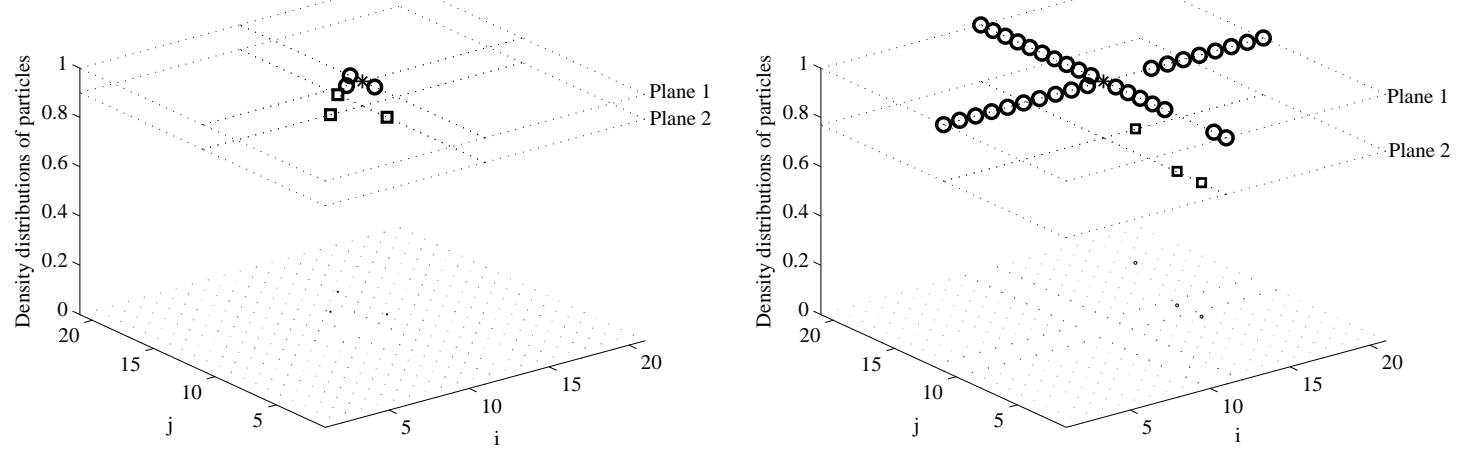

(a)

(b)

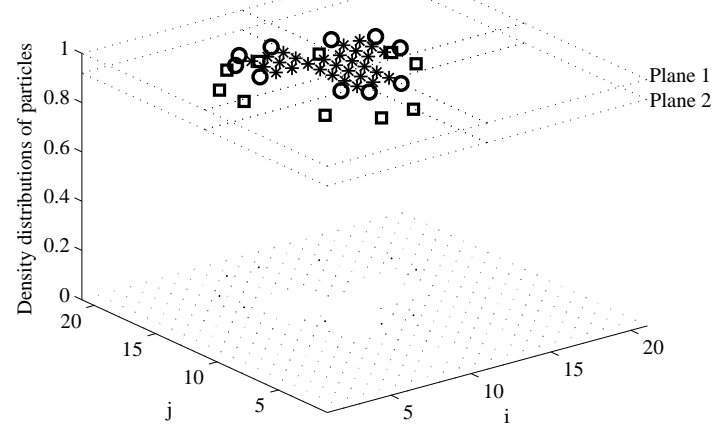

(c)

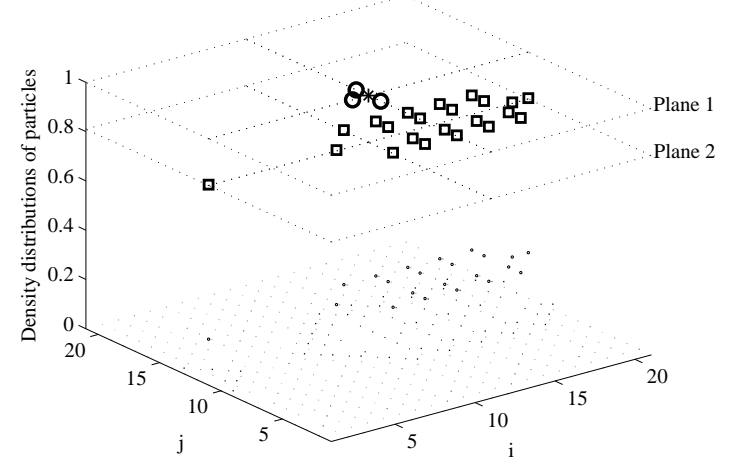

(d)

Fig.S3. Density plots of equilibrium structures formed at $\mathrm{T}=300 \mathrm{~K}$ for Case 2 at constant interaction energies (strong $=-3 \mathrm{eV}$, weak $=-1 \mathrm{eV}$ and rest all $=+1 \mathrm{eV}$ ) and varying particle concentrations. The equilibrium concentration of the A particle $(*)$ is $x_{\infty A}=0.0001$ for all plots, while the concentrations of $\mathrm{B}_{2}(\mathrm{O})$ and $\mathrm{C}$ particles $(\square)$ are varied as stated. (a) mixed T structure at $x_{\infty B_{2}}=0.0005$ and $x_{\infty C}=0.004$. (b) Chains of $\mathrm{B}_{2}$ particles at $x_{\infty B_{2}}=0.00153$ and $x_{\infty C}=0.004$. (c) Condensed A particles at $x_{\infty B_{2}}=0.0004$ and, $x_{\infty C}=0.004$. (d) Dimers of $\mathrm{C}$ particles at $x_{\infty B_{2}}=0.0025$ and $x_{\infty C}=0.008$. In all plots, Plane 1 contains $\mathrm{A}$ and $\mathrm{B}_{2}$ particles whereas Plane 2 contains only $\mathrm{C}$ particles. 